\title{
ON GROUPS WITH A TRIPLE FACTORISATION
}

\author{
by ANDREW FRANSMAN
}

(Received 20th December 1993)

Dedicated to Ismail J. Mohamed on his 65th birthday

\begin{abstract}
The aim of this paper is to discuss groups $G=H K=H A=K A$ with a triple factorisation as a product of two subgroups $H$ and $K$ and a nilpotent normal subgroup $A$. It is of interest to know whether such a group $G$ satisfies some nilpotency or supersolubility condition if $H$ and $K$ satisfy the same condition. A positive answer to this problem is given for certain group classes under the hypothesis that $A$ is prefactorised in $G=H K$. Some applications of the main theorem are also mentioned.
\end{abstract}

1991 Mathematics subject classification: 20F19.

\section{Introduction and statement of the main theorem}

Following Amberg and Höfling [9], a subgroup $S$ of a factorised group $G=H K$ is called prefactorised if $S=(H \cap S)(K \cap S)$, and if in addition $S$ contains $H \cap K$, then $S$ is said to be factorised (see Wielandt [20] or Amberg [1]). If $N$ is normal in $G=H K$, then the factoriser $X(N):=H N \cap K N$ can be written as follows:

$$
X(N)=N(H \cap K N)=N(K \cap H N)=(H \cap K N)(K \cap H N)
$$

see [1, Theorem 1.7 , p. 108]. Thus the investigation of factorised groups very often reduces to a triply factorised group $G=H K=H A=K A$, where $A \triangleleft G$; see [7]. Moreover, it is of interest to know whether $G$ belongs to a class of groups if $H$ and $K$ belong to the class and if the normal subgroup $A$ is nilpotent (see $[2,4,5,6,7,8$ and 11]).

The first two examples will provide some motivation for our main theorem.

Example 1. Let $S$ be a non-zero subring of the ring $\mathbb{C}$ of complex numbers under the usual operations. Consider the group

$$
G=G(S):=\left\{\left(\begin{array}{lll}
u & x & y \\
0 & u & z \\
0 & 0 & u
\end{array}\right) \mid u \in\langle-1\rangle, x, y, z \in S\right\},
$$


of $3 \times 3$ upper triangular matrices with respect to matrix multiplication. In short we write

$$
G=\left(\begin{array}{lll}
u & S & S \\
0 & u & S \\
0 & 0 & u
\end{array}\right)
$$

The following subgroups of $G$ are required:

and

$$
H=H(S):=\left(\begin{array}{lll}
u & 0 & S \\
0 & u & S \\
0 & 0 & u
\end{array}\right), \quad K=K(S):=\left(\begin{array}{lll}
u & S & S \\
0 & u & 0 \\
0 & 0 & u
\end{array}\right)
$$

$$
A=A(S):=\left(\begin{array}{lll}
1 & S & S \\
0 & 1 & S \\
0 & 0 & 1
\end{array}\right)
$$

It is easily checked that $G=H K=H A=K A$ and also that $A$ is prefactorised in $G=H K$. Furthermore $G^{\prime} \leqq A \cap \zeta(G)$, which implies that $A \triangleleft G$ and $G$ is nilpotent.

Example 2. Again let $S$ be a non-zero subring of the ring $\mathbb{C}$ of complex numbers under the usual operations. Consider the group

$$
G=G(S):=\left\{\left(\begin{array}{ll}
u & x \\
0 & v
\end{array}\right) \mid u, v \in\langle-1\rangle, x \in S\right\}
$$

of $2 \times 2$ upper triangular matrices with respect to matrix multiplication. (Note: for simplicity we will usually suppress $S$.) As in Example 1 we define the following subgroups of $G$ :

$$
U=\left(\begin{array}{ll}
1 & S \\
0 & 1
\end{array}\right), H=\left(\begin{array}{ll}
1 & S \\
0 & u
\end{array}\right), K=\left(\begin{array}{cc}
u & 0 \\
0 & v
\end{array}\right) \text { and } A=\left(\begin{array}{ll}
u & S \\
0 & u
\end{array}\right)
$$

It is readily seen that $G=H K=H A=K A$. Clearly $A$ is prefactorised in $G=H K$. Also $A=U \times \zeta(G)$ is abelian and normal in $G$. We now note:

(i) It is easy to show that $U$ is contained in the $F C$-centre of $H$ which in turn is properly contained in $H$. Now $U$ is maximal and of index 2 in $H$ and so $U$ is the $F C$ centre of $H$. Thus $H$ is $F C$-nilpotent of $F C$-class 2 . Obviously the Klein 4-group $K$ is $F C$-nilpotent. One can easily verify that $A$ is the $F C$-centre of $G=A K$. Consequently $G$ is $F C$-nilpotent of $F C$-class 2 , whence also $F C$-hypercentral and locally $F C$-nilpotent.

(ii) For each non-zero ideal $I$ of the ring $\mathbb{Z}$ of integers one can easily show that 
$G=G(I)$ is supersoluble, hence trivially, hypercyclic, locally supersoluble and locally polycyclic.

In both examples we note that $A \neq$ Fit $G$ and $A$ is not factorised in the Wielandt sense. This suggests our main result.

Theorem 1. Let $G=H K=H A=K A$ be the product of two subgroups $H$ and $K$ and $a$ nilpotent normal prefactorised subgroup $A$ of $G=H K$. If $H$ and $K$ belong to any of the following classes, then so does $G$.

(a) Nilpotent, hypercentral and locally nilpotent groups.

(b) FC-nilpotent, FC-hypercentral and locally FC-nilpotent groups.

(c) Finite-by-nilpotent groups.

(d) Hypercyclic, locally supersoluble and locally polycyclic groups.

It appears as if the prefactorised condition is a rather strong condition. Nevertheless, from the preceding discussion it is evident that we have an abundance of examples at our disposal. In fact, the groups $G=G(\mathbb{C})$ in both Example 1 and 2 satisfy the theorem, and so do (infinitely) many of their subgroups.

The prefactorised condition is also likely to occur in some finite groups as we demonstrate in the next example (see [9] for other cases).

Example 3. Let $p$ be an odd prime. Replace $\mathbb{C}$ in Example 1 by the finite field $\mathbb{Z}_{p}$ (under the usual operations). Then $G=G\left(\mathbb{Z}_{p}\right)=H K=H A=K A$, where $A=A\left(\mathbb{Z}_{p}\right)$ is normal and prefactorised in $G=H K=H\left(\mathbb{Z}_{p}\right) K\left(\mathbb{Z}_{p}\right)$, and $G$ is nilpotent.

We can also utilise Theorem 1 to provide simpler proofs for known results (see for instance Section 4: Applications, and especially Corollary 2).

If we remove the prefactorised condition, then the theorem becomes false in general. This is illustrated by an example due to Sysak [19], see Amberg [2].

Example 4. There exists a non-hypercentral group $G=A B=A M=B M$, where $A, B$ and $M$ are abelian and $M$ is normal, but not prefactorised in $G=A B$. Indeed, we have the following general construction of such a group (see [2; pp. 1-3]): Let $R$ be a radical ring and let $A$ be the set $R$ with operation

$$
r \circ s=r+s+r s \text { for every } r, s \in R \text {. }
$$

Then $A$ is a group which operates on the additive group $M=R^{+}$of $R$ via the rule

$$
m^{r}=m \circ r-r=m+m r \text { for every } m \in M, r \in A \text {. }
$$

Consider the semi-direct product $G=A \ltimes M=\{(r, m) \mid r \in A, m \in R\}$ with multiplication defined by 


$$
(r, s)\left(r^{\prime}, s^{\prime}\right)=\left(r+r^{\prime}+r r^{\prime}, s+s^{\prime}+s r^{\prime}\right)
$$

Identify the subgroups $\{(r, 0) \mid r \in A\},\{(0, m) \mid m \in R\} \subseteq R^{+}$and $\{(r, r) \mid r \in A\}$ of $G$ with $A, M$ and $B$ respectively. Then it can be shown that $G=A B=A M=B M$, where $A, B$ and $M$ are abelian and $(A \cap M)(B \cap M)=1=\zeta(G)$.

\section{Notation and preliminaries}

Our notation is standard (see [7], [17] or [18].

The terms of the lower central series of $G$ will be denoted by $\gamma_{r}(G)$ for $r>0$, with the usual convention that $\gamma_{2}(G)=G^{\prime}$, the derived group of $G$.

The terms of the upper central series of $G$ will be denoted by $\zeta_{r}(G)$ for $r \geqq 0$, with $\zeta_{1}(G)=\zeta(G)$, the centre of $G$.

Recall that the $F C$-centre $F_{1}(G)$ of a group $G$ is the set of all elements of $G$ which have only finitely many conjugates in $G$. The upper $F C$-central series of $G$ is defined by the rules:

$F_{0}(G)=1$,

$F_{\alpha+1}(G) / F_{\alpha}(G)=$ the $F C$-centre of $G / F_{\alpha}(G)$ for every ordinal $\alpha$,

$F_{\gamma}(G)=\bigcup_{\beta<\gamma} F_{\beta}(G)$ for limit ordinals $\gamma$.

A group $G$ is called $F C$-hypercentral if $F_{\tau}(G)=G$ for some ordinal $\tau$, and $G$ is said to be $F C$-nilpotent if $\tau$ is finite.

The following two lemmas will be needed in the sequel.

Lemma 1. Let $G=H K$ be factorised by $H$ and $K$, with $N$ a normal prefactorised subgroup of $G$. If $L \triangleleft G$, then $N L / L$ is prefactorised in $G / L=(H L / L)(K L / L)$.

Proof. The easy proof is left as an exercise.

Lemma 2. Let $G=H A$, where $H$ and $A$ are nilpotent subgroups and $A \triangleleft G$. If $N \triangleleft G$ and if $\gamma_{r}(N) \leqslant H \cap A$ for some positive integer $r$, then $N$ is nilpotent.

Proof. First of all assume that $A$ is abelian. We prove by induction on $t$ that $\gamma_{r+t}(N) \leqslant \gamma_{t+1}(H) \cap A$. The case $t=1$ is evident from the normality of $H \cap A$ in $G$. For $t>1$ it follows readily that $\gamma_{r+t}(N) \leqslant\left[\gamma_{r+t-1}(N), H\right] \leqslant \gamma_{t+1}(H) \cap A$, which completes the induction. The nilpotency of $H$ now clearly forces $N$ to be nilpotent. On the other hand, if $A$ is not abelian, then a routine check shows that $\gamma_{r}\left(N A^{\prime} / A^{\prime}\right) \leqslant\left(H A^{\prime} / A^{\prime}\right) \cap\left(A / A^{\prime}\right)$. Hence by the first part of the proof it is clear that $N A^{\prime} / A^{\prime}$ is nilpotent. By Fitting's Theorem [18; 5.2.8, p. 128] $N A / A^{\prime}$ is nilpotent. Now P. Hall's nilpotency criterion [18; 5.2 .10 , p. 129] yields $N A$ is nilpotent, whence so is $N$. 


\section{Proof of Theorem 1}

The proof of the theorem will be accomplished in a series of steps.

\subsection{Proof of Theorem 1(a).}

(i) The nilpotent case: By P. Hall's nilpotency criterion and Lemma 1, we may assume that $A$ is abelian. Thus $H \cap A$ and $K \cap A$ are normal in $G=H A=K A$. Clearly $G=K(H \cap A)$, which implies that $G /(H \cap A)$ is nilpotent. Consequently $G$ is nilpotent by Lemma 2.

(ii) The hypercentral case: By Robinson [16; Theorem 3, p. 228] and Lemma 1, we may assume that $A$ is abelian. Then the normal subgroup $H \cap A$ of $H A=G$ is centralised by $A$ and it is normalised by $H$. Now since $H$ is hypercentral, it follows that $H \cap A$ contains a non-trivial central element of $G$, which means that $\zeta_{1}(G) \neq 1$. By Lemma 1 the prefactorised property is inherited by every quotient group of $G$. Therefore $G$ is hypercentral.

(iii) The locally nilpotent case: In view of [16, Theorem 3, p. 230] and Lemma 1 we may assume that $A$ is abelian. It follows that $L=H \cap A$ is normal in $H A=G$. Since $G=K L$ we have that $G / L \cong K /(K \cap L)$ is locally nilpotent. Let $U$ be a finitely generated subgroup of $G$. Then $U L / L \cong U /(U \cap L)$ is finitely generated and hence nilpotent. So we may assume that $U \cap L \neq 1$. Therefore by [17, Part 1, Lemma 1.43, p. 32] there exists a finitely generated subgroup $E$ of $U \cap L$ such that $U \cap L=E^{U}$, because $U /(U \cap L)$ is finitely presented. Put $U=\left\langle u_{1}, \ldots, u_{i}\right\rangle$ and write $u_{i}=a_{i} h_{i}$, where $a_{i} \in A$ and $h_{i} \in H$. Consider the subgroups $H_{t}=\left\langle h_{1}, \ldots, h_{t}\right\rangle$ and $A_{t}=\left\langle a_{1}, \ldots, a_{t}\right\rangle$ of $U$. Since $L$ centralises $A$, it follows that $H_{t}$ normalises $U \cap L$. For each element $u \in U$ we obtain that $E^{u}=E^{h}$, for some $h \in H_{t}$. Now it is easily checked that

$$
U \cap L=E^{U} \leqslant E^{H_{t}} \leqslant U \cap L .
$$

This implies that

$$
U \cap L=E^{H_{t}} \leqslant\left\langle E, H_{t}\right\rangle \leqslant H
$$

Clearly $\left\langle E, H_{t}\right\rangle$ is nilpotent. Thus $\left\langle E, H_{t}\right\rangle$ satisfies the maximal condition on subgroups, whence $U \cap L$ is finitely generated. This implies that $M=\left\langle U \cap L, H_{t}\right\rangle$ is nilpotent. So since $A$ centralises the normal subgroup $U \cap L$ of $M$, there is a positive integer $c$ such that $\left[U \cap L,{ }_{c} M A\right]=1$. Now $U$ is clearly contained in $M A$. Therefore $U$ is nilpotent and hence $G$ is locally nilpotent.

\subsection{Proof of Theorem $\mathbf{1}(\mathbf{b})$.}

By virtue of [3, Proposition 3.1, p. 108] and Lemma 1 we may throughout this proof assume that $A$ is abelian.

(i) The $F C$-nilpotent case: Denote the upper $F C$-central series of $H$ by 


$$
1=\dot{F}_{0}(H) \leqslant F_{1}(H) \leqslant \cdots \leqslant F_{i}(H) \leqslant \cdots \leqslant F_{n}(H)
$$

Clearly we may assume that the normal subgroup $H \cap A$ of $H A=G$ is non-trivial. So by [17, Part 1, Lemma 2.16, p. 47] we have that $F_{1}(H) \cap A \neq 1$. Since $G=K(H \cap A)$ it follows that $G /(H \cap A)$ is $F C$-nilpotent. The fact that $A$ centralises each $F_{i}(H) \cap A$ implies that each $F_{i}(H) \cap A$ is normal in $G$. It is now easily checked that $G /\left(F_{i}(H) \cap A\right)$ is $F C$-nilpotent, for each $i=1, \ldots, n$. In particular, $G / F_{1}(H)$ is $F C$-nilpotent. It is also clear that $F_{1}(H) \cap A$ is contained in $F_{1}(G)$, whence $G / F_{1}(G)$ is $F C$-nilpotent. Therefore $G$ is $F C$-nilpotent.

(ii) The $F C$-hypercentral case: Analogously as in the $F C$-nilpotent case above one can show that $F_{1}(G) \neq 1$. Clearly by invoking Lemma 1 we conclude that $G$ is $F C$-hypercentral.

(iii) The locally $F C$-nilpotent case: Clearly $L=H \cap A$ is normal in $H A=G$. Furthermore, $G=K L$ and so $G / L \cong K /(K \cap L)$ is locally $F C$-nilpotent. Let $B$ be a finitely generated subgroup of $G$. Then $B L / L$ is $F C$-nilpotent and we infer from [13, Theorem 2, p. 40] that it is finitely generated nilpotent-by-finite. Moreover, it follows that $B /(B \cap L) \cong B L / L$ is finitely presented. Consequently there exists a finitely generated subgroup $E$ of $B \cap L$ such that $B \cap L=E^{B}$. Set $B=\left\langle b_{1}, \ldots, b_{t}\right\rangle$ and write $b_{i}=a_{i} h_{i}$, where $a_{i} \in A$ and $h_{i} \in H$. Consider the subgroups $H_{t}=\left\langle h_{1}, \ldots, h_{t}\right\rangle$ and $A_{t}=\left\langle a_{1}, \ldots, a_{t}\right\rangle$ of $B$. Then since $L$ centralises $A$, it follows that $H_{t}$ normalises $B \cap L$. For each $b \in B$ we now obtain that $E^{b}=E^{h}$, for some $h \in H_{r}$. Therefore

$$
B \cap L=E^{B} \leqslant E^{H_{t}} \leqslant B \cap L,
$$

and hence

$$
B \cap L=E^{H_{t}} \leqslant\left\langle E, H_{t}\right\rangle \leqslant H
$$

Since $H$ is locally $F C$-nilpotent, it again follows by [13, Theorem 2, p. 40] that the finitely generated subgroup $\left\langle E, H_{t}\right\rangle$ of $H$ is nilpotent-by-finite. This implies that $\left\langle E, H_{t}\right\rangle$ satisfies the maximal condition on subgroups. It follows that $B \cap L$ is finitely generated and so $M=\left\langle B \cap L, H_{t}\right\rangle$ is $F C$-nilpotent. Now since $B \cap L$ is normal in $M$, there exists an $F C$-central series $B \cap L=B_{1} \geqslant \cdots \geqslant B_{i} \geqslant \cdots \geqslant B_{n}=1$ of $M$. Obviously $A$ centralises $B \cap L$ and so this is also an $F C$-central series of $M A$. Moreover, since $B \subseteq M A$ it is apparent that $B \cap L$ is contained in some term of finite ordinal type of the upper $F C$ central series of $B$. Thus $B$ is $F C$-nilpotent and hence $G$ is locally $F C$-nilpotent.

\subsection{Proof of Theorem $1(c)$.}

The finite-by-nilpotent case: By [17, Part 1, Theorem 4.25, p. 117] and Lemma 1, we may assume that $A$ is abelian. Moreover, a further application of the aforementioned result from [17] yields that $\left|H: \zeta_{r}(H)\right|$ is finite, for some positive integer $r$. Obviously we may assume that $H \cap A \neq 1$. Suppose now that the normal subgroup $H \cap A$ of $H A=G$ is infinite. Then it has non-trivial intersection with $\zeta_{r}(H)$. This implies that $\zeta(H) \cap A \neq 1$. So 
it follows that $\zeta(G) \neq 1$ and we are done. On the other hand, if $H \cap A$ is finite, then $G /(H \cap A) \cong K /(K \cap H \cap A)$ is finite-by-nilpotent and so $G$ is finite-by-nilpotent.

\subsection{Proof of Theorem 1(d).}

(i) The hypercyclic case: By invoking [16, Theorem 2, p. 228] and Lemma 1 it may be assumed that $A$ is abelian. Consequently the normal subgroup $H \cap A$ of the hypercyclic group $H$ has an ascending $H$-invariant series with cyclic factors. However, since $A$ centralises the normal subgroup $H \cap A$ of $H A=G$ the series is indeed $G$-invariant. The fact that $G /(H \cap A) \cong K /(K \cap H \cap A)$ is hypercyclic finally forces $G$ to be hypercyclic.

(ii) The locally supersoluble case: Using [16, Theorem 3, p. 230] and Lemma 1, we may assume that $A$ is abelian. Let $U$ be a finitely generated subgroup of $G$. By exploring similar arguments as in the proof of the locally nilpotent case 3.1 (iii), one can show that the normal subgroup $U \cap H \cap A$ of $U$ has a finite $U$-invariant series with cyclic factors. However, the fact that $U /(U \cap H \cap A)$ is clearly supersoluble guarantees that $U$ is supersoluble. Thus $G$ is locally supersoluble.

(iii) The locally polycyclic case: Again by [16, Theorem 3, p. 230] and Lemma 1 we may assume that $A$ is abelian. Then it is obvious that $G /(H \cap A)$ is locally polycyclic. Let $U=\left\langle u_{1}, \ldots, u_{t}\right\rangle$ be any finitely generated subgroup of $G$. It follows that the factor group $U /(U \cap H \cap A)$ is polycyclic. So there are finitely many elements $v_{1}, \ldots, v_{s}$ such that

$$
U \cap H \cap A=\left\langle v_{1}, \ldots, v_{s}\right\rangle^{U}
$$

Put $u_{i}=h_{i} a_{i}$ where $h_{i} \in H$ and $a_{i} \in A \quad(1 \leqq i \leqq t)$. Now write $U_{t}=A H_{t}$, where $H_{t}=$ $\left\langle h_{1}, \ldots, h_{t}\right\rangle$. It follows that

$$
v_{i}^{U} \leqslant v_{i}^{U_{t}}=v_{i}^{H_{t}} \leqslant\left\langle v_{i}, h_{1}, \ldots, h_{t}\right\rangle \leqslant H,
$$

and hence every group $v_{i}^{U}$ is polycyclic. From this we conclude that $U \cap H \cap A$ is polycyclic. This implies that $U$ is polycyclic, in which case $G$ is locally polycyclic. This completes the proof of Theorem 1 .

\section{Applications}

We are in position to supply alternative proofs for two well-known results.

Corollary 1. (See [5,7 or 8]) If the finite group $G=H K=H A=K A$ is the product of nilpotent subgroups $H, K$ and $A$ with $A \triangleleft G$, then $G$ is nilpotent.

Proof. Write $F=$ Fit $G$ and observe that $G=H K=H F=K F$. We infer from [14, Corollary, p. 82] that $F$ is prefactorised in $G=H K$. So $G$ is nilpotent by Theorem 1 (a).

This is of course not the classical result of Kegel [12] (for that see Amberg and 
Fransman [8, Theorem A, p. 10]). Indeed, it is not clear to us how Theorem 1 could be used to derive Kegel's result.

However, in the final corollary we are able to give a surprisingly short proof, thereby avoiding quite heavy tools such as cohomology theory and splitting theorems for infinite groups (see [10]).

Corollary 2. (See [10, Lemma 4, p. 387]) Let $G$ be a minimax group and suppose that $G=H K=H A=K A$ where $H, K$ and $A$ are nilpotent and $A \triangleleft G$. Then $G$ is nilpotent.

Proof. Obviously $G=H K=H F=K F$, where $F=$ Fit $G$. Since $G$ is soluble we can apply [2, Theorem 5.1, p. 10] to obtain that $F$ is prefactorised in $G=H K$. But $F$ is contained in the Baer radical of $G$, which is nilpotent (see [15, Lemma 6.4, p. 46]). Therefore $F$ is nilpotent and hence $G$ is nilpotent by Theorem 1 (a).

Acknowledgement. Part of this work was done at the universities of Amsterdam and Mainz. The author wishes to express his sincere thanks to the mathematics departments of both institutions for splendid hospitality, and especially to Dr. R. W. van der Waall and Prof. B. Amberg for fruitful discussions and advice.

\section{REFERENCES}

1. B. Amberg, Artinian and noetherian factorized groups, Rend. Sem. Mat. Univ. Padova 55 (1976), 105-122.

2. B. Amberg, Triply factorized groups, in Groups St Andrews 1989 Volume 1 (C. M. Campbell and E. F. Robertson, eds.), (London Math. Soc. Lecture Notes Series 159, CUP Cambridge, New York, Port Chester, Melbourne, Sydney, 1991), pp. 1-13.

3. B. Amberg, S. Franciosi and F. de Grovanni, Groups with an FC-nilpotent triple factorization, Ricerche Mat. 36 (1987), 103-114.

4. B. Amberg, S. Franciosi and F. de Giovanni, Groups with a nilpotent triple factorisation, Bull. Austral. Math. Soc. 37 (1988), 69-79.

5. B. Amberg, S. Franciosi and F. de Giovanni, Groups with a supersoluble triple factorization, J. Algebra 117 (1988), 136-148.

6. B. Amberg, S. Franciosı and F. de GiovanNi, FC-nilpotent products of hypercentral groups, to appear.

7. B. Amberg, S. Franciosi and F. de Giovanni, Products of groups (Clarendon Press, Oxford, 1992). 9-18.

8. B. Amberg and A. Fransman, Products of groups and group classes, Israel J. Math. 87 (1994),

9. B. Amberg and B. Höfling, On finite products of nilpotent groups, Arch. Math. 63 (1994), $1-8$.

10. B. Amberg and D. J. S. Robinson, Soluble groups which are products of nilpotent minimax groups, Arch. Math. 42 (1984), 385-390.

11. A. Fransman, Factorizations of groups (Ph.D Thesis, Univ. of Amsterdam, The Netherlands, 1991). 
12. O. H. Kegel, Zur Struktur mehrfach faktorisierter endlicher Gruppen, Math. Z. 87 (1965), $42-48$.

13. D. H. Mclain, Remarks on the upper central series of a group, Proc. Glasgow Math. Assoc. 3 (1956), 38-44.

14. E. Pennington, On products of finite nilpotent groups, Math. Z. 134 (1973), 81-83.

15. D. J. S. Robinson, On the theory of subnormal subgroups, Math. Z. 89 (1965), 30-51.

16. D. J. S. Robinson, A property of the lower central series of a group, Math Z. 107 (1968), 225-231.

17. D. J. S. Robinson, Finiteness conditions and generalized soluble groups (Springer-Verlag, Berlin, Heidelberg, New York, 1972).

18. D. J. S. Robinson, $A$ course in the theory of groups (Springer-Verlag, New York, Heidelberg, Berlin, 1982).

19. Y. P. SYSAK, Products of infinite groups (preprint 82.53), Akad. Nauk. Ukr. Mat. Kiev (1982), $1-36$.

20. H. Wieland, Über Produkte von nilpotenten Gruppen, Illinois J. Math. 2 (1958), 611-618.

Department of Mathematics and Applied Mathematics,

UNIVERSity OF THE Western CAPE,

Private Bag X17,

7535 BeLlVILLE,

South Africa.

E-mail address: afransman@math.uwc.ac.za 